\title{
Exit Problems for Jump Processes Having Double-Sided Jumps with Rational Laplace Transforms
}

\author{
Yuzhen Wen and Chuancun Yin \\ School of Mathematical Sciences, Qufu Normal University, Qufu, Shandong 273165, China \\ Correspondence should be addressed to Chuancun Yin; ccyin@ mail.qfnu.edu.cn
}

Received 5 December 2013; Accepted 18 December 2013; Published 2 February 2014

Academic Editor: Xinguang Zhang

Copyright (C) 2014 Y. Wen and C. Yin. This is an open access article distributed under the Creative Commons Attribution License, which permits unrestricted use, distribution, and reproduction in any medium, provided the original work is properly cited.

\begin{abstract}
We consider the two-sided first-exit problem for a jump process having jumps with rational Laplace transform. We derive the joint distribution of the first passage time to two-sided barriers and the value of process at the first passage time. As applications, we present explicit expressions of the dividend formulae for barrier strategy and threshold strategy.
\end{abstract}

\section{Introduction}

Let $X=\{X(t): t \geq 0\}$ be a jump process defined on the filtered probability space $\left(\Omega, \mathscr{F}, \mathbb{F}=\mathscr{F}_{t}: t \geq 0, P\right)$ and

$$
X(t)=x+\mu t+\sum_{k=1}^{N(t)} Y_{k} \equiv x+\mu t+S(t), \quad t \geq 0
$$

where $x$ is the starting point of $X(t),\left\{N_{t} ; t \geq 0\right\}$ is a Poisson process with rate $\lambda$, constant $\mu \in R$ represents the drift, and the jump sizes $\left\{Y_{k} ; k \geq 1\right\}$ are independent and identically distributed random variables whose probability density function is given by $p(y)$. Moreover, it is assumed that $N_{t}$ and $Y_{k}$ are independent. Recently, one-sided and two-sided first-exit problems for processes with two-sided jumps have attracted a lot of attention in applied probability. For example, Perry et al. [1] studied two-sided first-exit time for processes with two-sided exponential jumps; Kou and Wang [2] studied the one-sided first passage times for a jump diffusion process with exponential positive and negative jumps. Cai [3] investigated the first passage time of a hyperexponential jump diffusion process. Chi [4] discussed the first passage time to two barriers of a hyperexponential jump diffusion process. Closed form expressions are obtained in [5] for the integral transforms of the joint distribution of the first-exit time from an interval and the value of the overshoot through boundaries at the exit time for the Poisson process with an exponential component. For some related works, see Perry et al. [6], Lewis and Mordecki [7], Kuznetsov [8],
Perry et al. [1], and the references therein. On the other hand, the jump processes with two-sided jumps have been studied widely in dividend problems. The problem of firstexit from a half-line is of fundamental interest with regard to the expected total discounted dividends up to ruin. See, for example, Chi [4], Chi and Lin [9], and Yin et al. [10].

Motivated by some related work mentioned above, we will assume $\left\{Y_{k}: k=1,2, \ldots\right\}$ is a sequence of iid random variables with a density $p(x)$ given by

$$
\begin{aligned}
p(x)= & \sum_{j=1}^{J} \sum_{i=1}^{m_{j}} p_{i j} \frac{\rho_{j}^{i} x^{i-1}}{(i-1) !} e^{-\rho_{j} x} \mathbf{1}_{\{x>0\}} \\
& +\sum_{j=1} \sum_{i=1}^{\widehat{m} \widehat{p}_{j}} \widehat{p}_{i j} \frac{\widehat{\rho}_{j}^{i}|x|^{i-1}}{(i-1) !} e^{\widehat{\rho}_{j} x} \mathbf{1}_{\{x<0\}} .
\end{aligned}
$$

We assume that $J, \widehat{J} \in N, m_{j}, \widehat{m}_{j} \in N, p_{i j}, \widehat{p}_{i j} \in R^{+}$, and $\sum_{j=1}^{J} \sum_{i=1}^{m_{j}} p_{i j}+\sum_{j=1}^{\widehat{J}} \sum_{i=1}^{\widehat{m}_{j}} \widehat{p}_{i j}=1, \operatorname{Re}\left(\rho_{j}\right)>0$ and $\operatorname{Re}\left(\widehat{\rho}_{j}\right)>0$, and that $\rho_{i} \neq \rho_{j}$ and $\hat{\rho}_{i} \neq \hat{\rho}_{j}$ for all $i \neq j$.

Define $\tau$ to be the first-exit time of $X_{t}$ to two flat barriers $a$ and $b(a<b)$; that is,

$$
\tau:=\inf \left\{t \geq 0: X_{t} \geq b \text { or } X_{t} \leq a\right\},
$$

and the infimum of an empty set is defined as $+\infty$.

The rest of the paper is organized as follows. In Section 2, we study the roots of the generalized Lundberg equation. 
Section 3 concentrates on deriving the joint distribution of the first-exit time and a nonnegative measurable function of the process value at the first-exit time to two flat barriers. In Section 4, we will study two kinds of dividend payment strategies by using the results in Section 3.

\section{Preliminary Results}

The infinitesimal generator of the jump process (1) is given by

$$
(L u)(x)=\mu u^{\prime}(x)+\lambda \int_{-\infty}^{\infty}[u(x+y)-u(x)] p(y) d y
$$

for any continuously differentiable function $u(x)$ and the Laplace exponent $G(z)=\ln E\left[\exp \left(z X_{1}\right)\right]$ is a rational function, which has the following partial fraction decomposition:

$$
G(z)=z \mu+\lambda\left(\sum_{j=1}^{J} \sum_{i=1}^{m_{j}} \frac{p_{i j}\left(\rho_{j}\right)^{i}}{\left(\rho_{j}-z\right)^{i}}+\sum_{j=1}^{\widehat{J}} \sum_{i=1}^{\widehat{m}_{j}} \frac{\widehat{p}_{i j}\left(\widehat{\rho}_{j}\right)^{i}}{\left(\hat{\rho}_{j}+z\right)^{i}}-1\right)
$$

We see that $G(z)$ has poles at $\rho_{j}(1 \leq j \leq J)$ and $-\widehat{\rho}_{j}(1 \leq$ $j \leq \widehat{J})$ with the corresponding multiplicities $m_{j}$ and $\widehat{m}_{j}$. Note that $G(z)$ has $\sum_{j=1}^{J} m_{j}$ poles (counting with multiplicity) in the half-plane $\operatorname{Re}(z)>0$ and $\sum_{j=1}^{\widehat{J}} \widehat{m}_{j}$ poles in the half-plane $\operatorname{Re}(z)<0$. We denote four marks:

(i) $M\{\widehat{M}\}$ is the number of poles of $G(z)$ in the halfplane $\operatorname{Re}(z)>0\{\operatorname{Re}(z)<0\}$,

(ii) $K\{\widehat{K}\}$ is the number of zeros of $G(z)-\theta$ in the halfplane $\operatorname{Re}(z)>0\{\operatorname{Re}(z)<0\}$.

In [8], the author has given the solutions of the CramérLundberg equation $G(z)=\theta$. However, for this simple Lévy process $X$, we will give another simple proof for the zeros of this equation.

Lemma 1. Assume that $\theta>0$.

(1) If $\mu>0$, then $K=M+1$ and $\widehat{K}=\widehat{M}$.

(2) If $\mu<0$, then $K=M$ and $\widehat{K}=\widehat{M}+1$.

(3) If $\mu=0$, then $K=M$ and $\widehat{K}=\widehat{M}$.

Proof. We denote

$$
\begin{gathered}
G_{1}(z)=z \mu-\lambda-\theta, \quad z \in C, \\
G_{2}(z)=\lambda\left(\sum_{j=1}^{J} \sum_{i=1}^{m_{j}} \frac{p_{i j}\left(\rho_{j}\right)^{i}}{\left(\rho_{j}-z\right)^{i}}+\sum_{j=1}^{\widehat{J}} \sum_{i=1}^{\widehat{m}_{j}} \frac{\widehat{p}_{i j}\left(\widehat{\rho}_{j}\right)^{i}}{\left(\hat{\rho}_{j}+z\right)^{i}}\right), \\
z \in C .
\end{gathered}
$$

Then $\left(\prod_{j=1}^{\widehat{J}}\left(\widehat{\rho}_{j}+z\right)^{\widehat{m}_{j}}\right) G_{1}(z)$ and $\left(\prod_{j=1}^{\widehat{J}}\left(\widehat{\rho}_{j}+z\right)^{\widehat{m}_{j}}\right) G_{2}(z)$ are analytic in $C^{-}$. Define a half circle $|x|=r, x \in C^{-}$, where $r>$ $\varepsilon+\max _{1 \leq j \leq \widehat{J}}\left\{\left|\widehat{\rho}_{j}\right|\right\}$, where $\varepsilon$ is an arbitrary positive constant. Note that $\left|G_{1}(z)\right| \rightarrow \infty$ for $\operatorname{Re}(z) \rightarrow-\infty$ and $\left|G_{2}(z)\right| \leq$ $\lambda \sum_{j=1}^{J} \sum_{i=1}^{m_{j}}\left(\left|p_{i j}\right|\left|\rho_{j}\right|^{i} / \varepsilon^{i}\right)+\lambda \sum_{j=1}^{\widehat{J}} \sum_{i=1}^{\widehat{m}_{j}}\left(\left|\widehat{p}_{i j}\right|\left|\widehat{\rho}_{j}\right|^{i} / \varepsilon^{i}\right)$ is bounded for $\operatorname{Re}(z) \rightarrow-\infty$. When $r$ is large enough,

$$
\left|\left(\prod_{j=1}^{\widehat{J}}\left(\hat{\rho}_{j}+z\right)^{\widehat{m}_{j}}\right) G_{1}(z)\right|>\left|\left(\prod_{j=1}^{\widehat{I}}\left(\hat{\rho}_{j}+z\right)^{\widehat{m}_{j}}\right) G_{2}(z)\right|
$$

on the boundary of the half circle in $C^{-}$. On the imaginary axis, we observe that

$$
\left|G_{1}(i a)\right|=|\lambda+\theta+i a \mu|>\lambda=\left|G_{2}(i a)\right|, \quad a \in R .
$$

Since $\left(\prod_{j=1}^{\widehat{J}}\left(\widehat{\rho}_{j}+z\right)^{\widehat{m}_{j}}\right) G_{1}(z)$ has $\widehat{M}$ roots with negative real part, so the equation $G(z)=\theta, \theta>0$, has $\widehat{M}+1$ roots with negative real part according to Rouchés theorem. Similarly, we can prove that $G(z)=\theta, \theta>0$ has $M$ roots with positive real part. The proofs of (2) and (3) are similar to the proof of (1).

Remark 2. In [8], the author states that it is very unlikely for the equation $G(z)=\theta$ to have multiple solutions, so we assume all the roots of this equation are distinct. We denote $\gamma_{i}(\theta)=\gamma_{i}(i=1,2, \ldots, M+1)$ and $\widehat{\gamma}_{i}(\theta)=\widehat{\gamma}_{i}(i=$ $1,2, \ldots, \widehat{M}+1)$ for notational simplicity.

\section{Distribution of the First-Exit Time to Two Flat Barriers}

In this section, we consider the distribution of the firstexit time to two flat barriers. Let $k, J, m_{j}, \widehat{J}, \widehat{m}_{j^{\prime}}$ be positive integers. Suppose $I_{1 k}=(1,1, \ldots, 1)_{1 \times k}, k=M+1$, $\widehat{M}+1, E_{1 \widehat{M}}=\left(e^{\widehat{r}_{1}(b-a)}, \ldots, e^{\widehat{r}_{\widehat{M}}(b-a)}\right)_{1 \times \widehat{M}}$, and $E_{1 M}=$ $\left(e^{r_{1}(b-a)}, \ldots, e^{r_{M}(b-a)}\right)_{1 \times M}$. For $i=M, M+1$ and $j=\widehat{M}, \widehat{M}+1$, define

$$
A_{11 i}=\left(\begin{array}{ccc}
\frac{\rho_{1}}{\rho_{1}-\gamma_{1}} & \cdots & \frac{\rho_{1}}{\rho_{1}-\gamma_{i}} \\
\vdots & \ddots & \vdots \\
\left(\frac{\rho_{1}}{\rho_{1}-\gamma_{1}}\right)^{m_{1}} & \cdots & \left(\frac{\rho_{1}}{\rho_{1}-\gamma_{i}}\right)^{m_{1}} \\
\vdots & \ddots & \vdots \\
\frac{\rho_{J}}{\rho_{J}-\gamma_{1}} & \cdots & \frac{\rho_{J}}{\rho_{J}-\gamma_{i}} \\
\vdots & \ddots & \vdots \\
\left(\frac{\rho_{J}}{\rho_{J}-\gamma_{1}}\right)^{m_{J}} & \cdots & \left(\frac{\rho_{J}}{\rho_{J}-\gamma_{i}}\right)^{m_{J}}
\end{array}\right),
$$




$$
\begin{aligned}
& A_{12 j}=\left(\begin{array}{ccc}
\frac{e^{\widehat{r}_{1}(b-a)} \rho_{1}}{\rho_{1}-\widehat{\gamma}_{1}} & \cdots & \frac{e^{\widehat{r}_{\mathcal{M}}(b-a)} \rho_{1}}{\rho_{1}-\widehat{\gamma}_{j}} \\
\vdots & \ddots & \vdots \\
\frac{e^{\widehat{r}_{1}(b-a)} \rho_{1}^{m_{1}}}{\left(\rho_{1}-\widehat{\gamma}_{1}\right)^{m_{1}}} & \cdots & \frac{e^{\widehat{T}_{\widehat{M}}(b-a)} \rho_{1}^{m_{1}}}{\left(\rho_{1}-\widehat{\gamma}_{j}\right)^{m_{1}}} \\
\vdots & \ddots & \vdots \\
\frac{e^{\widehat{r}_{1}(b-a)} \rho_{J}}{\rho_{J}-\widehat{\gamma}_{1}} & \cdots & \frac{e^{\widehat{r}_{\widehat{M}}(b-a)} \rho_{J}}{\rho_{J}-\widehat{\gamma}_{j}} \\
\vdots & \ddots & \vdots \\
\frac{e^{\widehat{r}_{1}(b-a)} \rho_{J}^{m_{J}}}{\left(\rho_{J}-\widehat{\gamma}_{1}\right)^{m_{J}}} & \cdots & \frac{e^{\widehat{r}_{\widehat{M}}(b-a)} \rho_{J}^{m_{J}}}{\left(\rho_{J}-\widehat{\gamma}_{j}\right)^{m_{J}}}
\end{array}\right)
\end{aligned}
$$

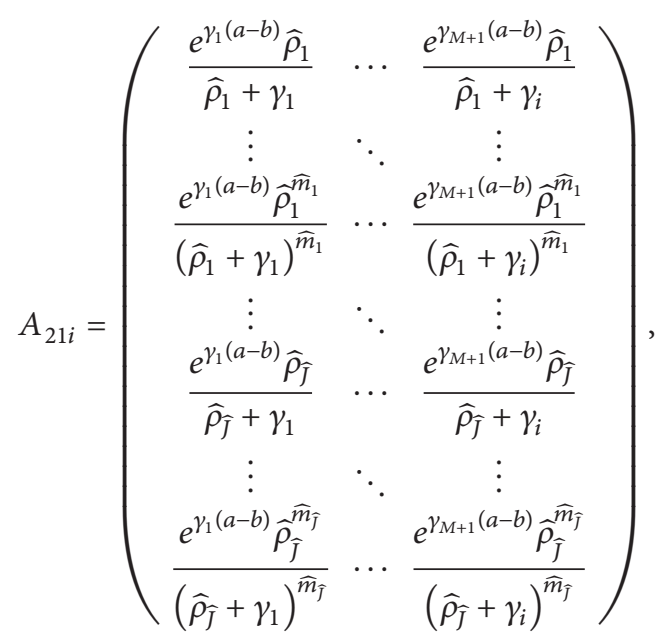

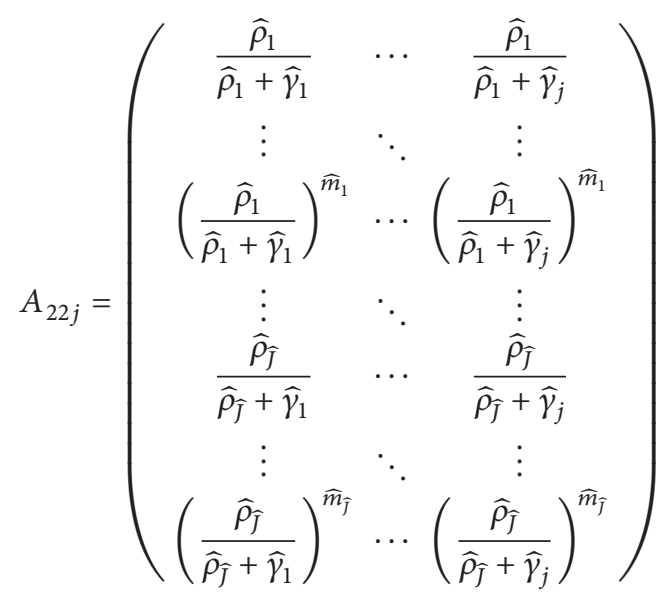

$$
\begin{aligned}
& A_{1}=\left(\begin{array}{cc}
I_{1 M+1} & E_{1 \times \widehat{M}} \\
A_{11 M+1} & A_{12 \widehat{M}} \\
A_{21 M+1} & A_{22 \widehat{M}}
\end{array}\right), \quad A_{2}=\left(\begin{array}{cc}
A_{11 M} & A_{12 \widehat{M}+1} \\
E_{1 M} & I_{1 \widehat{M}+1} \\
A_{21 M} & A_{22 \widehat{M}+1}
\end{array}\right), \\
& A_{3}=\left(\begin{array}{ll}
A_{11 M} & A_{12 \widehat{M}} \\
A_{21 M} & A_{22 \widehat{M}}
\end{array}\right) .
\end{aligned}
$$

Note that $A_{1}$ and $A_{2}$ are $(M+\widehat{M}+1) \times(M+\widehat{M}+1)$ matrices and $A_{3}$ is an $(M+\widehat{M}) \times(M+\widehat{M})$ matrix.
Theorem 3. Consider any nonnegative measurable function $f$ such that

$$
\begin{gathered}
f_{j k}^{u}=\int_{0}^{\infty} f(y+b) \frac{\left(\rho_{j} y\right)^{k-1} \rho_{j} e^{-\rho_{j} y}}{(k-1) !} d y<\infty, \\
f_{j^{\prime} k^{\prime}}^{d}=\int_{-\infty}^{0} f(y+a) \frac{\left(-\widehat{\rho}_{j} y\right)^{k^{\prime}-1} \widehat{\rho}_{j} e^{\widehat{\rho}_{j} y}}{\left(k^{\prime}-1\right) !} d y<\infty
\end{gathered}
$$

for $j=1,2, \ldots, J, k=1, \ldots, m_{j}, j^{\prime}=1, \ldots, \widehat{J}, k^{\prime}=1, \ldots, \widehat{m}_{j^{\prime}}$. For any $\theta>0$ and $u \in(a, b)$, one has the following.

(1) If $\mu>0$, then

$$
E^{u}\left[e^{-\theta \tau} f\left(X_{\tau}\right)\right]=\pi_{1} \mathbf{f}_{1} .
$$

Here

$$
\begin{gathered}
\pi_{1}=\left(E^{u}\left[e^{-\theta \tau} \mathbf{1}_{K_{0}}\right], E^{u}\left[e^{-\theta \tau} \mathbf{1}_{K_{11}}\right], \ldots,\right. \\
E^{u}\left[e^{-\theta \tau} \mathbf{1}_{K_{J, m_{J}}}\right], E^{u}\left[e^{-\theta \tau} \mathbf{1}_{G_{11}}\right], \ldots, \\
E^{u}\left[e^{-\theta \tau} \mathbf{1}_{\left.\left.\bar{G}_{\tilde{T}, \tilde{m}_{\tilde{J}}}\right]\right)^{T},}\right. \\
f_{1}=\left(f(b), f_{11}^{u}, \ldots, f_{j k}^{u}, \ldots, f_{J_{m_{J}}}^{u}, f_{11}^{d}, \ldots, f_{j^{\prime} k^{\prime}}^{d}, \ldots, f_{\tilde{J} \tilde{m}_{\tilde{T}}}^{d}\right)^{T}
\end{gathered}
$$

are both $M+\widehat{M}+1$-dimensional vectors, and $\pi_{1}$ satisfies

$$
\pi_{1} A_{1}=\varpi_{1},
$$

where $\Phi_{1}=\left(e^{\gamma_{1}(u-b)}, e^{\gamma_{2}(u-b)}, \ldots, e^{\gamma_{M+1}(u-b)}, e^{\widehat{\gamma}_{1}(u-a)}, e^{\widehat{\gamma}_{2}(u-a)}\right.$, $\left.\ldots, e^{{\widehat{\gamma_{M}}}_{M}(u-a)}\right)$ is an $M+\widehat{M}+1$-dimensional vector. Moreover, when $A_{1}$ is a nonsingular matrix, $\pi_{1}$ is the unique solution of (13); that is,

$$
\pi_{1}=\omega_{1} \cdot A_{1}^{-1}
$$

(2) If $\mu<0$, then

$$
E^{u}\left[e^{-\theta \tau} f\left(X_{\tau}\right)\right]=\pi_{2} \mathbf{f}_{2} .
$$

Here $\pi_{2}=\left(E^{u}\left[e^{-\theta \tau} \mathbf{1}_{K_{11}}\right], \ldots, E^{u}\left[e^{-\theta \tau} \mathbf{1}_{K_{J, m_{J}}}\right], E^{u}\left[e^{-\theta \tau} \mathbf{1}_{G_{0}}\right]\right.$, $E^{u}\left[e^{-\theta \tau} \mathbf{1}_{G_{11}}\right], \ldots, E^{u}\left[e^{-\theta \tau} \mathbf{1}_{\left.G_{\hat{T}, \tilde{m}_{\tilde{T}}}\right]}\right)^{T}$ and $f_{2}=\left(f_{11}^{u}, \ldots, f_{j k}^{u}, \ldots\right.$, $\left.f_{J m_{J}}^{u}, f(a), f_{11}^{d}, \ldots, f_{j^{\prime} k^{\prime}}^{d}, \ldots, f_{\widehat{J} \widehat{m}_{\widehat{J}}}^{d}\right)^{T}$ are both $M+\widehat{M}+1-$ dimensional vectors, and $\pi_{2}$ satisfies

$$
\pi_{2} A_{2}=\omega_{2},
$$

where $\omega_{2}=\left(e^{\gamma_{1}(u-b)}, e^{\gamma_{2}(u-b)}, \ldots, e^{\gamma_{M}(u-b)}, e^{\widehat{\gamma}_{1}(u-a)}, e^{\widehat{\gamma}_{2}(u-a)}\right.$, ..., $\left.e^{\widehat{\gamma}_{\bar{M}+1}(u-a)}\right)$ is an $M+\widehat{M}+1$-dimensional vector. Moreover, when $A_{2}$ is a nonsingular matrix, $\pi_{2}$ is the unique solution of (16); that is,

$$
\pi_{2}=\omega_{2} \cdot A_{2}^{-1} .
$$


(3) If $\mu=0$, then

$$
E^{u}\left[e^{-\theta \tau} f\left(X_{\tau}\right)\right]=\pi_{3} \mathbf{f}_{3} .
$$

Here $\pi_{3}=\left(E^{u}\left[e^{-\theta \tau} \mathbf{1}_{K_{11}}\right], \ldots, E^{u}\left[e^{-\theta \tau} \mathbf{1}_{K_{J, m J}}\right], E^{u}\left[e^{-\theta \tau} \mathbf{1}_{G_{11}}\right], \ldots\right.$, $E^{u}\left[e^{-\theta \tau} \mathbf{1}_{\left.G_{\mathcal{T}_{T, m_{T}}}\right]}\right)^{T}$ and $f_{3}=\left(f_{11}^{u}, \ldots, f_{j k}^{u}, \ldots, f_{J m_{J}}^{u}, f_{11}^{d}, \ldots\right.$, $\left.f_{j^{\prime} k^{\prime}}^{d}, \ldots, f_{\widehat{J} \widehat{m}_{\widehat{T}}}^{d}\right)^{T}$ are both $M+\widehat{M}$-dimensional vectors, and $\pi_{3}$ satisfies

$$
\pi_{3} A_{3}=\omega_{3}
$$

where $\Phi_{3}=\left(e^{\gamma_{1}(u-b)}, e^{\gamma_{2}(u-b)}, \ldots, e^{\gamma_{M}(u-b)}, e^{\widehat{\gamma}_{1}(u-a)}, e^{\widehat{\gamma}_{2}(u-a)}, \ldots\right.$, $\left.e^{\widehat{\gamma}_{\widehat{M}}(u-a)}\right)$ is an $M+\widehat{M}$-dimensional vector. Moreover, when $A_{3}$ is a nonsingular matrix, $\pi_{3}$ is the unique solution of (19); that is,

$$
\pi_{3}=\omega_{3} \cdot A_{3}^{-1}
$$

Proof. When the jump process $X$ exits the band $(a, b)$, sometimes it hits the boundary exactly and sometimes it jumps across the boundaries when leaving. We introduce a sequence of events:

$$
\begin{aligned}
& K_{0}:=\left\{\omega: X_{\tau}=b\right\}, G_{0}:=\left\{\omega: X_{\tau}=a\right\}, \\
& K_{j i l}:=\left\{\omega: X_{(\cdot)} \text { crosses } b \text { at time } \tau \text { by the } l\right. \text { th phase of } \\
& \left.i \text { th positive jump whose parameter is } \rho_{j}\right\}, \\
& G_{j^{\prime} i^{\prime} l^{\prime}}:=\left\{\omega: X_{(\cdot)} \text { crosses } a \text { at time } \tau \text { by the } l^{\prime}\right. \text { th phase } \\
& \text { of } \left.i^{\prime} \text { th negative jump whose parameter is } \widehat{\rho}_{j^{\prime}}\right\} \\
& K_{j k}=K_{j k 1} \cup K_{j(k+1) 2} \cup \cdots \cup K_{j m_{j}\left(m_{j}-k+1\right)}, \\
& G_{j^{\prime} k^{\prime}}=G_{j^{\prime} k^{\prime} 1} \cup G_{j^{\prime}\left(k^{\prime}+1\right) 2} \cup \cdots \cup G_{j^{\prime} m_{j}^{\prime}\left(m_{j}^{\prime}-k^{\prime}+1\right)} \\
& \text { for } j=1,2, \ldots, J, j^{\prime}=1,2, \ldots, \widehat{J}, i=1, \ldots, m_{i}, i^{\prime}= \\
& 1, \ldots, \widehat{m}_{i}, l=1, \ldots, i \text {, and } l^{\prime}=1, \ldots, i^{\prime} .
\end{aligned}
$$

It is easy to check that

$$
M_{t}=e^{-\theta t+\beta X_{t}}-e^{\beta X_{0}}-(G(z)-\theta) \int_{0}^{t} e^{-\theta s+\beta X_{s}} d s, \quad t \geq 0
$$

is a zero-mean martingale for every $\theta>0$ and every $\beta \in C$. We apply the sampling theorem to the martingale $M=\left(M_{t} \mid\right.$ $t \geq 0$ ) and get $E^{\mathcal{u}}\left[M_{\tau}\right]=E^{\mathcal{u}}\left[M_{0}\right]=0$; that is,

$$
E^{u}\left[e^{-\theta \tau+\beta X_{\tau}}\right]-e^{\beta X_{0}}=(G(z)-\theta) E^{u}\left[\int_{0}^{\tau} e^{-\theta s+\beta X_{s}} d s\right] .
$$

Inserting the roots of $G(z)=\theta$ in (22), we get that the right side of (22) becomes zero.

From Perry et al. [1], we know that for $l=1,2, \ldots, i, i=$ $1,2, \ldots, m_{j}$, and $j=1,2, \ldots, J$, given $K_{j i l}$, the overshoot $X_{\tau}-b$ is independent of $\tau$ and Erlang $(i+j-l)$ distributed with scale parameter $\rho_{j}$ and for $l^{\prime}=1, \ldots, i^{\prime}, i^{\prime}=1, \ldots, \widehat{m}_{i}$, and $j^{\prime}=1,2, \ldots, \widehat{J}$, given $G_{j^{\prime} i^{\prime} l^{\prime}}$, the excess $a-X_{\tau}$ is independent of $\tau$ and Erlang $\left(i^{\prime}+j^{\prime}-l^{\prime}\right)$ distributed with scale parameter $\widehat{\rho}_{j^{\prime}}$. Therefore, we know that, for $j=1,2, \ldots, J$ and $k=$
$1,2, \ldots, m_{j}$, given $K_{j k}$, the overshoot $X_{\tau}-b$ is independent of $\tau$ and Erlang $(k)$ distributed with scale parameter $\rho_{j}$ and for $j^{\prime}=1,2, \ldots, \widehat{J}$ and $k^{\prime}=1,2, \ldots, \widehat{m}_{j^{\prime}}$, given $G_{j^{\prime} k^{\prime}}$, the excess $a-X_{\tau}$ is independent of $\tau$ and $\operatorname{Erlang}\left(k^{\prime}\right)$ distributed with scale parameter $\hat{\rho}_{j^{\prime}}$.

If $\mu>0$, by the law of total probability, we have

$$
\begin{aligned}
E^{u}\left[e^{-\theta \tau} f\left(X_{\tau}\right)\right] & E^{u}\left[e^{-\theta \tau} f\left(X_{\tau}\right) \mathbf{1}_{K_{0}}\right]+\sum_{j=1}^{J} \sum_{i=1}^{m_{j}} \sum_{l=1}^{i} E^{u}\left[e^{-\theta \tau} f\left(X_{\tau}\right) \mathbf{1}_{K_{j i l}}\right] \\
& +\sum_{j^{\prime}=1}^{\widehat{J}} \sum_{i^{\prime}=1}^{\widehat{m}_{j^{\prime}}} \sum_{l^{\prime}=1}^{i^{\prime}} E^{u}\left[e^{-\theta \tau} f\left(X_{\tau}\right) \mathbf{1}_{G_{j^{\prime} i^{\prime} l^{\prime}}}\right] \\
= & E^{u}\left[e^{-\theta \tau} f\left(X_{\tau}\right) \mathbf{1}_{K_{0}}\right]+\sum_{j=1}^{J} \sum_{k=1}^{m_{j}} E^{u}\left[e^{-\theta \tau} f\left(X_{\tau}\right) \mathbf{1}_{K_{j k}}\right] \\
& +\sum_{j^{\prime}=1}^{\widehat{J}} \sum_{k^{\prime}=1}^{\widehat{m}_{j^{\prime}}} E^{u}\left[e^{-\theta \tau} f\left(X_{\tau}\right) \mathbf{1}_{G_{j^{\prime} k^{\prime}}}\right] \\
= & E^{u}\left[e^{-\theta \tau} \mathbf{1}_{K_{0}}\right] f(b)+\sum_{j=1}^{J} \sum_{k=1}^{m_{j}} E^{u}\left[e^{-\theta \tau} \mathbf{1}_{K_{j k}}\right] f_{j k}^{u} \\
& +\sum_{j^{\prime}=1}^{\widehat{J}} \sum_{k^{\prime}=1}^{\widehat{m}_{j^{\prime}}} E^{u}\left[e^{-\theta \tau} \mathbf{1}_{G_{j^{\prime} k^{\prime}}}\right] f_{j^{\prime} k^{\prime}}^{d} .
\end{aligned}
$$

Inserting $\gamma_{i}, i=1,2, \ldots, M+1$ and $\widehat{\gamma}_{i}, i=1,2, \ldots, \widehat{M}$, in (22), it follows that, for $i=1,2, \ldots, M+1$,

$$
\begin{aligned}
e^{\gamma_{i} u}= & E^{u}\left[e^{-\theta \tau+\gamma_{i} X_{\tau}}\right] \\
= & E^{u}\left[e^{-\theta \tau} \mathbf{1}_{K_{0}}\right] e^{\gamma_{i} b} \\
& +\sum_{j=1}^{J} \sum_{k=1}^{m_{j}} E^{u}\left[e^{-\theta \tau} \mathbf{1}_{K_{j k}}\right]\left(\frac{\rho_{j}}{\rho_{j}-\gamma_{i}}\right)^{k} e^{\gamma_{i} b} \\
& +\sum_{j^{\prime}=1}^{\widehat{J}} \sum_{k^{\prime}=1}^{\widehat{m}_{j^{\prime}}} E^{u}\left[e^{-\widehat{\rho} \tau} \mathbf{1}_{G_{j^{\prime} k^{\prime}}}\right]\left(\frac{\widehat{\rho}_{j^{\prime}}}{\widehat{\rho}_{j^{\prime}}+\gamma_{i}}\right)^{k^{\prime}} e^{\gamma_{i} a}
\end{aligned}
$$

and for $i=1,2, \ldots, \widehat{M}$

$$
\begin{aligned}
e^{\widehat{\gamma_{i}} u}= & E^{u}\left[e^{-\theta \tau+\widehat{\gamma}_{i} X_{\tau}}\right] \\
= & E^{u}\left[e^{-\theta \tau} \mathbf{1}_{K_{0}}\right] e^{\widehat{\gamma}_{i} b} \\
& +\sum_{j=1}^{J} \sum_{k=1}^{m_{j}} E^{u}\left[e^{-\theta \tau} \mathbf{1}_{K_{j k}}\right]\left(\frac{\rho_{j}}{\rho_{j}-\widehat{\gamma}_{i}}\right)^{k} e^{\widehat{\gamma}_{i} b} \\
& +\sum_{j^{\prime}=1}^{\widehat{J}} \sum_{k^{\prime}=1}^{\widehat{m}_{j^{\prime}}} E^{u}\left[e^{-\widehat{\rho} \tau} \mathbf{1}_{G_{j^{\prime} k^{\prime}}}\right]\left(\frac{\widehat{\rho}_{j^{\prime}}}{\widehat{\rho}_{j^{\prime}}+\widehat{\gamma}_{i}}\right)^{k^{\prime}} e^{\widehat{\gamma}_{i} a} .
\end{aligned}
$$


Then the vector $\pi$ satisfies $\pi_{1} \cdot A_{1}=\Phi_{1}$ and we have (11). If $A_{1}$ is nonsingular, we have $\pi_{1}=\varrho_{1} A_{1}^{-1}$.

The proof of (2) and (3) is similar and we omit their proofs.

Corollary 4. If the matrix $D$ is nonsingular, then for any $\delta \in\left(-\min \left(\left|\widehat{\rho}_{1}\right|,\left|\hat{\rho}_{2}\right|, \ldots,\left|\widehat{\rho}_{\hat{\jmath}}\right|\right), \min \left(\left|\rho_{1}\right|,\left|\rho_{2}\right|, \ldots,\left|\rho_{J}\right|\right)\right)$, one has the following.

(1) If $\mu>0$, then

$$
\begin{array}{r}
E^{u}\left[e^{-\theta \tau+\delta X_{\tau}}\right]=e^{\delta b} \cdot\left(\sum_{i=1}^{M+1} \omega_{i} e^{\gamma_{i}(u-b)}+\sum_{j=1}^{\widehat{M}} v_{j} e^{\widehat{\gamma}_{j}(u-a)}\right), \\
a<u<b,
\end{array}
$$

where

$$
\begin{gathered}
A_{1} \cdot\left(\omega_{1}, \omega_{2}, \ldots, \omega_{M+1}, v_{1}, v_{2}, \ldots, v_{\widehat{M}}\right)^{T}=J_{1}(\delta), \\
J_{1}(\delta)=\left(1,\left(\frac{\rho_{1}}{\rho_{1}-\delta}\right)^{m_{1}}, \ldots, \frac{\rho_{1}}{\rho_{1}-\delta}, \ldots,\left(\frac{\rho_{I}}{\rho_{J}-\delta}\right)^{m_{J}}, \ldots,\right. \\
\left.\quad \frac{\rho_{J}}{\rho_{J}-\delta},\left(\frac{\widehat{\rho}_{1}}{\widehat{\rho}_{1}+\delta}\right)^{\widehat{m}_{1}} e^{\delta(a-b)}, \ldots, \frac{\widehat{\rho}_{\widehat{J}}}{\widehat{\rho}_{\widehat{J}}+\delta} e^{\delta(a-b)}\right)^{T} .
\end{gathered}
$$

(2) If $\mu<0$, then

$$
\begin{array}{r}
E^{u}\left[e^{-\theta \tau+\delta X_{\tau}}\right]=e^{\delta b} \cdot\left(\sum_{i=1}^{M} \bar{\omega}_{i} e^{\gamma_{i}(u-b)}+\sum_{j=1}^{\widehat{M}+1} \bar{v}_{j} e^{\widehat{\gamma}_{j}(u-a)}\right), \\
a<u<b,
\end{array}
$$

where

$$
\begin{array}{r}
A_{2} \cdot\left(\bar{\omega}_{1}, \bar{\omega}_{2}, \ldots, \bar{\omega}_{M}, \bar{\nu}_{1}, \bar{\nu}_{2}, \ldots, \bar{v}_{\widehat{M}+1}\right)^{T}=J_{2}(\delta), \\
J_{2}(\delta)=\left(\left(\frac{\rho_{1}}{\rho_{1}-\delta}\right)^{m_{1}}, \ldots, \frac{\rho_{1}}{\rho_{1}-\delta}, \ldots,\left(\frac{\rho_{J}}{\rho_{J}-\delta}\right)^{m_{J}},\right. \\
\ldots, \frac{\rho_{J}}{\rho_{J}-\delta}, e^{\delta(a-b)}, \\
\left.\left(\frac{\widehat{\rho}_{1}}{\hat{\rho}_{1}+\delta}\right)^{\widehat{m}_{1}} e^{\delta(a-b)}, \ldots, \frac{\widehat{\rho}_{\widehat{J}}}{\widehat{\rho}_{\widehat{J}}+\delta} e^{\delta(a-b)}\right)^{T} .
\end{array}
$$

(3) If $\mu=0$, then

$$
\begin{array}{r}
E^{u}\left[e^{-\theta \tau+\delta X_{\tau}}\right]=e^{\delta b} \cdot\left(\sum_{i=1}^{M+1} \widetilde{\omega}_{i} e^{\gamma_{i}(u-b)}+\sum_{j=1}^{\widehat{M}+1} \widetilde{\nu}_{j} e^{\widehat{\gamma}_{j}(u-a)}\right), \\
a<u<b,
\end{array}
$$

where

$$
\begin{gathered}
A_{3} \cdot\left(\widetilde{\omega}_{1}, \widetilde{\omega}_{2}, \ldots, \widetilde{\omega}_{M+1}, \widetilde{v}_{1}, \widetilde{v}_{2}, \ldots, \widetilde{v}_{\widehat{M}+1}\right)^{T}=J_{3}(\delta), \\
J_{3}(\delta)=\left(\left(\frac{\rho_{1}}{\rho_{1}-\delta}\right)^{m_{1}}, \ldots, \frac{\rho_{1}}{\rho_{1}-\delta}, \ldots,\left(\frac{\rho_{I}}{\rho_{I}-\delta}\right)^{m_{J}}, \ldots,\right. \\
\left.\quad \frac{\rho_{I}}{\rho_{I}-\delta},\left(\frac{\widehat{\rho}_{1}}{\widehat{\rho}_{1}+\delta}\right)^{\widehat{m}_{1}} e^{\delta(a-b)}, \ldots, \frac{\widehat{\rho}_{\hat{I}}}{\widehat{\rho}_{\widehat{J}}+\delta} e^{\delta(a-b)}\right)^{T} .
\end{gathered}
$$

Corollary 5. For any $\theta>0, u, b \in R$, one has the following. (1) If $\mu>0$, then

$$
E^{u}\left[e^{-\theta \tau^{b}} f\left(X_{\tau^{b}}\right)\right]= \begin{cases}f(u), & u \geq b ; \\ \sum_{l=1}^{M+1} \omega_{l} e^{\gamma_{l}(u-b)}, & u<b .\end{cases}
$$

Here $\omega=\left(\omega_{1}, \omega_{2}, \ldots, \omega_{M+1}\right)^{T}$ is a vector uniquely determined by the following linear system:

$$
D_{1} \omega=\bar{f}_{1},
$$

where $\bar{f}_{1}=\left(f(b), f_{11}^{u}, \ldots, f_{1 m_{1}}^{u}, \ldots, f_{J 1}^{u}, \ldots, f_{J m_{J}}^{u}\right)$ is an $(M+$ 1)-dimensional vector and $D_{1}$ is an $(M+1) \times(M+1)$ nonsingular matrix:

$D_{1}$

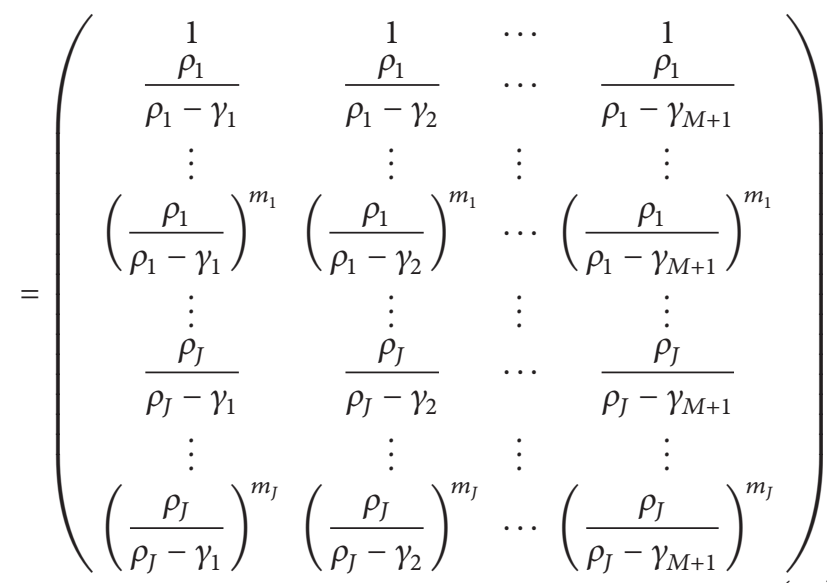

(2) If $\mu \leq 0$, then

$$
E^{u}\left[e^{-\theta \tau^{b}} f\left(X_{\tau^{b}}\right)\right]= \begin{cases}f(u), & u \geq b ; \\ \sum_{l=1}^{M} \bar{\omega}_{l} e^{\gamma_{l}(u-b)}, & u<b .\end{cases}
$$

Here $\bar{\omega}=\left(\bar{\omega}_{1}, \bar{\omega}_{2}, \ldots, \bar{\omega}_{M}\right)^{T}$ is a vector uniquely determined by the following linear system:

$$
D_{2} \bar{\omega}=\bar{f}_{2},
$$


where $\bar{f}_{2}=\left(f_{11}^{u}, \ldots, f_{1 m_{1}}^{u}, \ldots, f_{J 1}^{u}, \ldots, f_{J m_{J}}^{u}\right)$ is an $M$ dimensional vector and $D_{2}$ is an $M \times M$ nonsingular matrix:

$D_{2}$

$$
=\left(\begin{array}{cccc}
\frac{\rho_{1}}{\rho_{1}-\gamma_{1}} & \frac{\rho_{1}}{\rho_{1}-\gamma_{2}} & \cdots & \frac{\rho_{1}}{\rho_{1}-\gamma_{M+1}} \\
\vdots & \vdots & \vdots & \vdots \\
\left(\frac{\rho_{1}}{\rho_{1}-\gamma_{1}}\right)^{m_{1}} & \left(\frac{\rho_{1}}{\rho_{1}-\gamma_{2}}\right)^{m_{1}} & \cdots & \left(\frac{\rho_{1}}{\rho_{1}-\gamma_{M+1}}\right)^{m_{1}} \\
\vdots & \vdots & \vdots & \vdots \\
\frac{\rho_{I}}{\rho_{J}-\gamma_{1}} & \frac{\rho_{I}}{\rho_{I}-\gamma_{2}} & \cdots & \frac{\rho_{J}}{\rho_{J}-\gamma_{M+1}} \\
\vdots & \vdots & \vdots & \vdots \\
\left(\frac{\rho_{J}}{\rho_{J}-\gamma_{1}}\right)^{m_{J}} & \left(\frac{\rho_{I}}{\rho_{J}-\gamma_{2}}\right)^{m_{J}} & \cdots & \left(\frac{\rho_{J}}{\rho_{J}-\gamma_{M+1}}\right)^{m_{J}}
\end{array}\right) .
$$

Corollary 6. For any $\theta>0, u, a \in R$, one has the following. (1) If $\mu>0$, then

$$
E^{u}\left[e^{-\theta \tau_{a}} f\left(X_{\tau_{a}}\right)\right]= \begin{cases}f(u), & u \leq a ; \\ \sum_{l=1}^{\widehat{M}} \omega_{l} e^{\gamma_{l}(u-a)}, & u>a .\end{cases}
$$

Here $v=\left(v_{1}, v_{2}, \ldots, v_{\widehat{M}}\right)^{T}$ is a vector uniquely determined by the following linear system:

$$
C_{1} v=\tilde{f}_{1},
$$

where $\tilde{f}_{1}=\left(f_{11}^{d}, \ldots, f_{1 \widehat{m}_{1}}^{d}, \ldots, f_{\widehat{I}_{1}}^{d}, \ldots, f_{\widehat{J}_{\widehat{T}}}^{d}\right)$ is an $\widehat{M}$ dimensional vector and $C_{1}$ is an $(\widehat{M}) \times(\widehat{M})$ nonsingular matrix: $C_{1}$

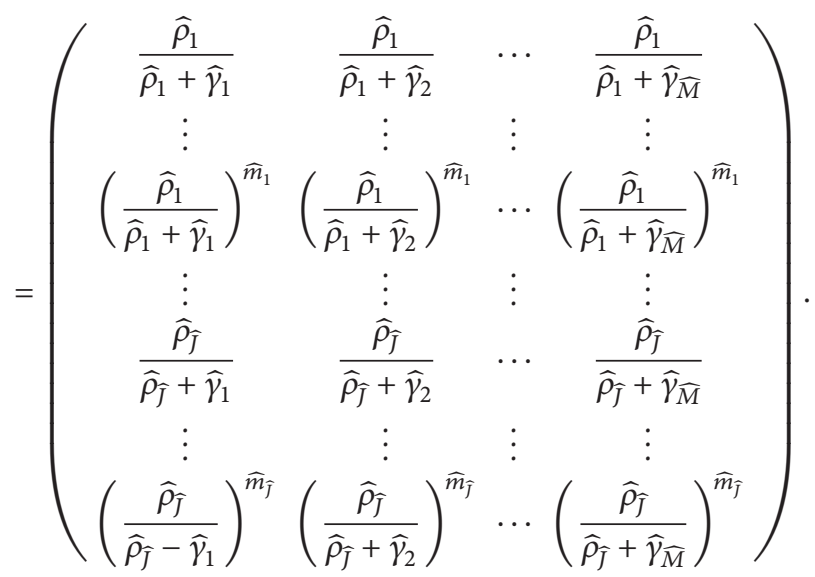

(2) If $\mu \leq 0$, then

$$
E^{u}\left[e^{-\theta \tau_{a}} f\left(X_{\tau_{a}}\right)\right]= \begin{cases}f(u), & u \leq a ; \\ \sum_{l=1}^{\widehat{M}+1} \bar{\omega}_{l} e^{\bar{\nu}_{l}(u-a)}, & u<a .\end{cases}
$$

Here $\bar{\nu}=\left(\bar{\nu}_{1}, \bar{\nu}_{2}, \ldots, \bar{\nu}_{\widehat{M}+1}\right)^{T}$ is a vector uniquely determined by the following linear system:

$$
C_{2} \bar{v}=\tilde{f}_{2},
$$

where $\widetilde{f}_{2}=\left(f(a), f_{11}^{d}, \ldots, f_{1 \widehat{m}_{1}}^{d}, \ldots, f_{\widehat{J} 1}^{d}, \ldots, f_{\widehat{J} \widehat{m}_{\widehat{T}}}^{d}\right)$ is an $\widehat{M}+1$ dimensional vector and $C_{2}$ is an $(\widehat{M}+1) \times(\widehat{M}+1)$ nonsingular matrix:

$\mathrm{C}_{2}$

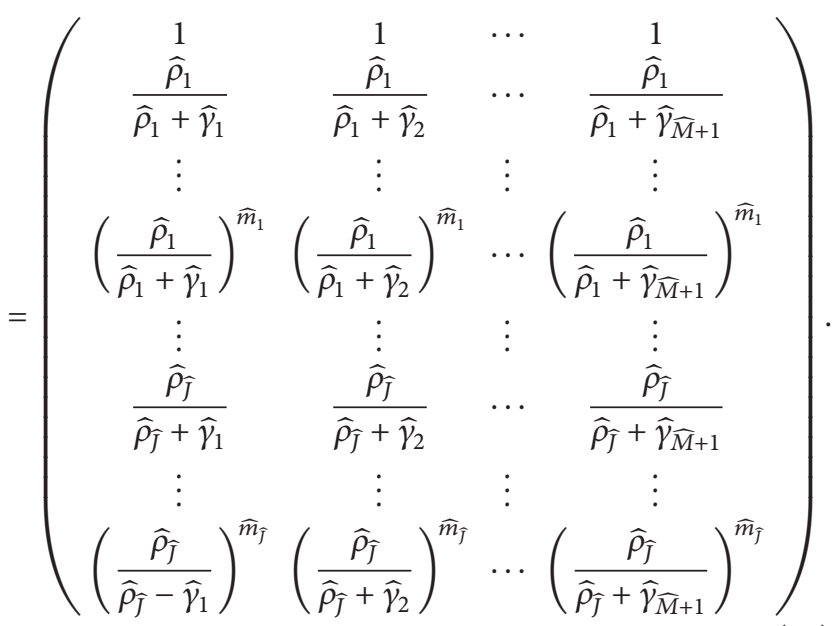

Example 7. When $J=m_{1}=\widehat{J}=\widehat{m}_{1}=1, p(x)=$ $p_{11} \rho_{1} e^{-\rho_{1} x} I_{(x>0)}+\widehat{p}_{11} \hat{\rho}_{1} e^{\hat{\rho}_{1} x} I_{(x<0)}$, and $p_{11}+\widehat{p}_{11}=1, \mu>0$, then the equation $G(z)=\theta$ has 3 real roots: $\gamma_{1}, \gamma_{2}$, and $\widehat{\gamma}_{1}$ $\left(-\infty<\widehat{\gamma}_{1}<0<\gamma_{1}<\gamma_{2}<\infty\right)$. Let

$$
A_{1}=\left(\begin{array}{ccc}
1 & 1 & e^{\widehat{\gamma}_{1}(b-a)} \\
\frac{\rho_{1}}{\rho_{1}-\gamma_{1}} & \frac{\rho_{1}}{\rho_{1}-\gamma_{2}} & \frac{\rho_{1} e^{\widehat{\gamma}_{1}(b-a)}}{\rho_{1}-\widehat{\gamma}_{1}} \\
\frac{\hat{\rho}_{1} e^{\gamma_{1}(a-b)}}{\widehat{\rho}_{1}+\gamma_{1}} & \frac{\widehat{\rho}_{1} e^{\gamma_{2}(a-b)}}{\hat{\rho}_{1}+\gamma_{2}} & \frac{\widehat{\rho}_{1}}{\widehat{\rho}_{1}+\widehat{\gamma}_{1}}
\end{array}\right) .
$$

Denote $A_{1}^{-1}$ by

$$
A_{1}^{-1}=\frac{1}{\left|A_{1}\right|}\left(\begin{array}{ccc}
a_{11} & -a_{21} & a_{31} \\
-a_{12} & a_{22} & -a_{32} \\
a_{13} & -a_{23} & a_{33}
\end{array}\right) .
$$

Then we have

$$
E^{u}\left[e^{-\theta \tau} f\left(X_{\tau}\right)\right]=\omega_{1} e^{\gamma_{1}(u-b)}+\omega_{2} e^{\gamma_{2}(u-b)}+\omega_{3} e^{\widehat{\gamma}_{1}(u-a)},
$$

where

$$
\begin{gathered}
\omega_{1}=\frac{a_{11} f(b)-a_{21} f_{11}^{u}+a_{31} f_{11}^{d}}{\left|A_{1}\right|}, \\
\omega_{2}=\frac{-a_{12} f(b)+a_{22} f_{11}^{u}-a_{32} f_{11}^{d}}{\left|A_{1}\right|}, \\
\omega_{3}=\frac{a_{13} f(b)-a_{23} f_{11}^{u}+a_{33} f_{11}^{d}}{\left|A_{1}\right|}, \\
f_{11}^{u}=\int_{0}^{\infty} f(y+b) \rho_{1} e^{-\rho_{1} y} d y,
\end{gathered}
$$




$$
\begin{aligned}
& f_{11}^{d}=\int_{-\infty}^{0} f(y+a) \hat{\rho}_{1} e^{\hat{\rho}_{1} y} d y, \\
& \left|A_{1}\right|=\frac{\rho_{1} \hat{\rho}_{1}\left(\gamma_{2}-\gamma_{1}\right)}{\left(\rho_{1}-\gamma_{1}\right)\left(\rho_{1}-\gamma_{2}\right)\left(\hat{\rho}_{1}+\widehat{\gamma}_{1}\right)} \\
& +\frac{\rho_{1} \widehat{\rho}_{1}\left(\gamma_{1}-\widehat{\gamma}_{1}\right) e^{\left(\gamma_{2}-\widehat{\gamma}_{1}\right)(a-b)}}{\left(\rho_{1}-\gamma_{1}\right)\left(\rho_{1}-\widehat{\gamma}_{1}\right)\left(\hat{\rho}_{1}+\gamma_{2}\right)} \\
& +\frac{\rho_{1} \widehat{\rho}_{1}\left(\gamma_{1}-\widehat{\gamma}_{2}\right) e^{\left(\gamma_{1}-\widehat{\gamma}_{1}\right)(a-b)}}{\left(\rho_{1}-\gamma_{2}\right)\left(\left(\rho_{1}-\widehat{\gamma}_{1}\right)\right)\left(\widehat{\rho}_{1}+\gamma_{1}\right)}, \\
& a_{11}=\frac{\rho_{1} \hat{\rho}_{1}}{\left(\rho_{1}-\gamma_{2}\right)\left(\widehat{\rho}_{1}+\widehat{\gamma}_{1}\right)}-\frac{\rho_{1} \widehat{\rho}_{1} e^{\left(\gamma_{2}-\widehat{\gamma}_{1}\right)(a-b)}}{\left(\rho_{1}-\widehat{\gamma}_{1}\right)\left(\hat{\rho}_{1}+\gamma_{2}\right)}, \\
& a_{21}=\frac{\widehat{\rho}_{1}}{\hat{\rho}_{1}+\widehat{\gamma}_{1}}-\frac{\hat{\rho}_{1} e^{\left(\gamma_{2}-\widehat{\gamma}_{1}\right)(a-b)}}{\widehat{\rho}_{1}+\gamma_{2}}, \\
& a_{13}=\frac{\rho_{1} \widehat{\rho}_{1} e^{\gamma_{2}(a-b)}}{\left(\rho_{1}-\gamma_{1}\right)\left(\hat{\rho}_{1}+\gamma_{2}\right)}-\frac{\rho_{1} \widehat{\rho}_{1} e^{\gamma_{1}(a-b)}}{\left(\rho_{1}-\gamma_{2}\right)\left(\hat{\rho}_{1}+\gamma_{1}\right)}, \\
& a_{23}=\frac{\rho_{1} e^{\gamma_{2}(a-b)}}{\hat{\rho}_{1}+\gamma_{2}}-\frac{\widehat{\rho}_{1} e^{\gamma_{1}(a-b)}}{\hat{\rho}_{1}+\gamma_{1}}, \quad a_{31}=\frac{\rho\left(\widehat{\gamma}_{1}-\gamma_{2}\right) e^{\widehat{\gamma}(b-a)}}{\left(\rho_{1}-\widehat{\gamma}_{1}\right)\left(\widehat{\rho}_{1}-\gamma_{2}\right)}, \\
& a_{33}=\frac{\rho\left(\gamma_{1}-\gamma_{2}\right)}{\left(\rho_{1}-\gamma_{1}\right)\left(\widehat{\rho}_{1}-\gamma_{2}\right)} \text {. }
\end{aligned}
$$

We define $a_{12}\left(a_{22}, a_{32}\right)$ as follows: let $a_{12}\left(a_{22}, a_{32}\right)$ be obtained from $a_{11}\left(a_{21}, a_{31}\right)$ by changing $\gamma_{2}$ to $\gamma_{1}$ in $a_{11}\left(a_{21}, a_{31}\right)$.

(i) If $f\left(X_{\tau}\right)=e^{\delta X_{\tau}}$, then we have

$$
\begin{array}{r}
E^{u}\left[e^{-\theta \tau+\delta X_{\tau}}\right]=e^{\delta b}\left(\omega_{1}^{\prime} e^{\gamma_{1}(u-b)}+\omega_{2}^{\prime} e^{\gamma_{2}(u-b)}+\omega_{3}^{\prime} e^{\widehat{\gamma}_{1}(u-a)}\right), \\
a<u<b,
\end{array}
$$

where

$$
\begin{gathered}
f(b)=e^{\delta b}, \quad f_{11}^{u}=e^{\delta b} \frac{\rho_{1}}{\rho_{1}-\delta}, \quad f_{11}^{d}=e^{\delta a} \frac{\hat{\rho}_{1}}{\hat{\rho}_{1}+\delta}, \\
\omega_{1}^{\prime}=\frac{a_{11}-a_{21}\left(\rho_{1} /\left(\rho_{1}-\delta\right)\right)+a_{31}\left(\widehat{\rho}_{1} e^{\delta(a-b)} /\left(\widehat{\rho}_{1}+\delta\right)\right)}{\left|A_{1}\right|} \\
\omega_{2}^{\prime}=\frac{-a_{12}+a_{22}\left(\rho_{1} /\left(\rho_{1}-\delta\right)\right)-a_{32}\left(\widehat{\rho}_{1} e^{\delta(a-b)} /\left(\hat{\rho}_{1}+\delta\right)\right)}{\left|A_{1}\right|}, \\
\omega_{3}^{\prime}=\frac{a_{13}-a_{23}\left(\rho_{1} /\left(\rho_{1}-\delta\right)\right)+a_{33}\left(\hat{\rho}_{1} e^{\delta(a-b)} /\left(\hat{\rho}_{1}+\delta\right)\right)}{\left|A_{1}\right|} .
\end{gathered}
$$

(ii) If $f\left(X_{\tau}\right)=I_{\left(X_{\tau}-b \geq 0\right)}$, then we have

$$
\begin{array}{r}
E^{u}\left[e^{-\theta \tau} I_{\left(X_{\tau}-b \geq 0\right)}\right]=\widehat{\omega}_{1} e^{\gamma_{1}(u-b)}+\widehat{\omega}_{2} e^{\gamma_{2}(u-b)}+\widehat{\omega}_{3} e^{\widehat{\gamma}_{1}(u-a),} \\
a<u<b,
\end{array}
$$

where

$$
f(b)=1, \quad f_{11}^{u}=1, \quad f_{11}^{d}=0,
$$

$\widehat{\omega}_{1}=\frac{a_{11}-a_{21}}{\left|A_{1}\right|}, \quad \widehat{\omega}_{2}=\frac{-a_{12}+a_{22}}{\left|A_{1}\right|}, \quad \widehat{\omega}_{3}=\frac{a_{13}-a_{23}}{\left|A_{1}\right|}$.

(iii) If $f\left(X_{\tau}\right)=I_{\left(X_{\tau}-a \leq 0\right)}$, then we have

$$
\begin{array}{r}
E^{u}\left[e^{-\theta \tau} I_{\left(X_{\tau}-a \leq 0\right)}\right]=\widehat{\widehat{\omega}}_{1} e^{\gamma_{1}(u-b)}+\widehat{\hat{\omega}}_{2} e^{\gamma_{2}(u-b)}+\widehat{\omega}_{3} e^{\widehat{\gamma}_{1}(u-a)}, \\
a<u<b,
\end{array}
$$

where

$$
\begin{array}{cc}
f(b)=0, \quad f_{11}^{u}=0, & f_{11}^{d}=1, \\
\widehat{\widehat{\omega}}_{1}=\frac{a_{31}}{\left|A_{1}\right|}, \quad \widehat{\hat{\omega}}_{2}=\frac{-a_{32}}{\left|A_{1}\right|}, \quad \widehat{\hat{\omega}}_{3}=\frac{a_{33}}{\left|A_{1}\right|} .
\end{array}
$$

(iv) If $f\left(X_{\tau}\right)=I_{\left(X_{\tau}-b>y\right)}, y>0$, then we have

$$
\begin{aligned}
& E^{u}\left[e^{-\theta \tau} I_{\left(X_{\tau}-b>y\right)}\right] \\
&=\check{\check{\omega}}_{1} e^{\gamma_{1}(u-b)}+\check{\check{\omega}}_{2} e^{\gamma_{2}(u-b)}+\check{\check{\omega}}_{3} e^{\widehat{\gamma}_{1}(u-a)}, \\
& a<u<b,
\end{aligned}
$$

where

$$
f(b)=0, \quad f_{11}^{u}=e^{-\rho_{1} y}, \quad f_{11}^{d}=0,
$$

$\check{\omega}_{1}=-\frac{a_{21} e^{-\rho_{1} y}}{\left|A_{1}\right|}, \quad \check{\omega}_{2}=\frac{a_{22} e^{-\rho_{1} y}}{\left|A_{1}\right|}, \quad \check{\omega}_{3}=\frac{-a_{23} e^{-\rho_{1} y}}{\left|A_{1}\right|}$.

(v) If $f\left(X_{\tau}\right)=I_{\left(X_{\tau}-a<-y\right)}, y>0$, then we have

$$
\begin{array}{r}
E^{u}\left[e^{-\theta \tau} I_{\left(X_{\tau}-a<-y\right)}\right]=\check{\check{\omega}}_{1} e^{\gamma_{1}(u-b)}+\check{\check{\omega}}_{2} e^{\gamma_{2}(u-b)}+\check{\check{\omega}}_{3} e^{\widehat{\gamma}_{1}(u-a),} \\
a<u<b,
\end{array}
$$

where

$$
\begin{gathered}
f(b)=0, \quad f_{11}^{u}=0, \quad f_{11}^{d}=e^{-\widehat{\rho}_{1} y}, \\
\check{\check{\omega}}_{1}=\frac{-a_{31} e^{-\widehat{\rho}_{1} y}}{\left|A_{1}\right|}, \quad \check{\check{\omega}}_{2}=\frac{a_{32} e^{-\widehat{\rho}_{1} y}}{\left|A_{1}\right|}, \quad \check{\check{\omega}}_{3}=\frac{-a_{33} e^{-\widehat{\rho}_{1} y}}{\left|A_{1}\right|} .
\end{gathered}
$$

(vi) If $f\left(X_{\tau}\right)=I_{\left(X_{\tau}=b\right)}$, then we have

$$
\begin{aligned}
E^{u} & {\left[e^{-\theta \tau} I_{\left(X_{\tau}=b\right)}\right] } \\
& =\widetilde{\omega}_{1} e^{\gamma_{1}(u-b)}+\widetilde{\omega}_{2} e^{\gamma_{2}(u-b)}+\widetilde{\omega}_{3} e^{{\widehat{\gamma_{1}}}^{(u-a)},}
\end{aligned}
$$


where

$$
\begin{gathered}
f(b)=1, \quad f_{11}^{u}=0, \quad f_{11}^{d}=0, \quad \widetilde{\omega}_{1}=\frac{a_{11}}{\left|A_{1}\right|}, \\
\widetilde{\omega}_{2}=\frac{-a_{12}}{\left|A_{1}\right|}, \quad \widetilde{\omega}_{3}=\frac{a_{13}}{\left|A_{1}\right|} .
\end{gathered}
$$

\section{Dividend Problems}

In this section, we are going to derive dividend formulae for barrier strategy and threshold strategy, based on the results obtained in Section 3.

For barrier strategy, it is assumed that dividends are paid according to a barrier strategy $b$. Such a strategy has a level of the barrier $b>0$; when the surplus exceeds the barrier, the excess is paid out immediately as the dividend. The modified surplus at time $t$ is given by

$$
U(t)=X(t)-D(t), \quad t \geq 0,
$$

where $D(t)$ denote the aggregate dividends paid between time 0 and time $t$; that is,

$$
D(t)=\left(\max _{0 \leq s \leq t} X(s)-b\right)^{+} .
$$

Let

$$
T=\inf \{t \geq 0: U(t)<0\}
$$

be the time of ruin for the modified surplus $U$ and

$$
D=\int_{0}^{T} e^{-\theta t} d D(t)
$$

the present value of all dividends until ruin, where $\theta>0$ is the discount factor.

Denote by $V(u ; b)$ the expectation of the discounted dividends until ruin, if the barrier strategy with parameter $b$ is applied; that is,

$$
V(u ; b)=E[D \mid X(0)=u], \quad 0 \leq u \leq b .
$$

Note that we have

$$
V(u ; b)=u-b+V(b ; b), \quad u>b .
$$

Theorem 8. Let $\tau_{b}^{+}=\inf \{t \geq 0: X(t) \geq b\}$ and $\tau=T \wedge \tau_{b}^{+}$. Then for $\theta>0$, one has the following.

(1) for $0<u<b$,

$$
\begin{aligned}
V(u ; b)= & \sum_{j=1}^{J} \sum_{i=1}^{m_{j}} \frac{m_{j}+1-i}{\eta_{j}} E^{u}\left[e^{-\theta \tau_{b}^{+}} \mathbf{1}_{K_{j i}}\right] \\
& +V(b, b)\left(E^{u}\left[e^{-\theta \tau_{b}^{+}} \mathbf{1}_{K_{0}}\right]\right. \\
& \left.+\sum_{j=1}^{J} \sum_{i=1}^{m_{j}} E^{u}\left[e^{-\theta \tau_{b}^{+}} \mathbf{1}_{K_{j i}}\right]\right) ;
\end{aligned}
$$

$$
\text { (2) if } \mu>0 \text {, then }
$$

$V(b ; b)$

$$
=\frac{\left.\sum_{j=1}^{J} \sum_{i=1}^{m_{j}}\left(\left(m_{j}+1-i\right) / \eta_{j}\right)\left(E^{u}\left[e^{-\theta \tau_{b}^{+}} \mathbf{1}_{K_{j i}}\right]\right)^{\prime}\right|_{u=b}}{\left.\left(E^{u}\left[e^{-\theta \tau_{b}^{+}} \mathbf{1}_{K_{0}}\right]+\sum_{j=1}^{J} \sum_{i=1}^{m_{j}} E^{u}\left[e^{-\theta \tau_{b}^{+}} \mathbf{1}_{K_{j i}}\right]\right)^{\prime}\right|_{u=b}}
$$

(3) if $\mu \leq 0$, then

$$
V(b ; b)=\frac{\sum_{j=1}^{J} \sum_{i=1}^{m_{j}}\left(\left(m_{j}+1-i\right) / \eta_{j}\right) E^{b}\left[e^{-\theta \tau_{b}^{+}} \mathbf{1}_{K_{j i}}\right]}{1-\sum_{j=1}^{J} \sum_{i=1}^{m_{j}} E^{u}\left[e^{-\theta \tau_{b}^{+}} \mathbf{1}_{K_{j i}}\right]} .
$$

Proof. It follows from the conditional memoryless property, the conditional independence, and the strong Markov property that, for $0 \leq u \leq b$, we have

$$
\begin{aligned}
& V(u ; b)=E^{u}\left[\int_{0}^{\tau} e^{-\theta t} d D(t)\right]=E^{u}\left[\int_{0}^{\tau} e^{-\theta t} d D(t) \mathbf{1}_{\left\{\tau_{b}^{+}<T\right\}}\right] \\
& =E^{u}\left[\left(X_{\tau}-b+V(b ; b)\right) e^{-\theta \tau_{b}^{+}} \mathbf{1}_{\left\{\tau_{b}^{+}<T\right\}} \mathbf{1}_{\left\{X_{\tau}>b\right\}}\right] \\
& +E^{u}\left[\int_{\tau_{b}^{+}}^{T} e^{-\theta t} d D(t) \mathbf{1}_{\left\{\tau_{b}^{+}<T\right\}} \mathbf{1}_{\left\{X_{\tau}=b\right\}}\right] \\
& =\sum_{j=1}^{J} \sum_{i=1}^{m_{j}} E^{u}\left[\left(X_{\tau_{b}^{+}}-b\right) e^{-\theta \tau_{b}^{+}} \mathbf{1}_{K_{j i}}\right] \\
& +V(b ; b) \sum_{j=1}^{J} \sum_{i=1}^{m_{j}} E^{u}\left[e^{-\theta \tau_{b}^{+}} \mathbf{1}_{K_{j i}}\right] \\
& +E^{u}\left[e^{-\theta \tau_{b}^{+}} \mathbf{1}_{\left\{\tau_{b}^{+}<T\right\}} \mathbf{1}_{\left\{X_{\tau}=b\right\}} E\right. \\
& \left.\times\left(\int_{0}^{T} e^{-\theta t} d D(t) \cdot \theta_{\tau_{b}^{+}} \mid \mathscr{F}_{\tau_{b}^{+}}^{X}\right)\right] \\
& =\sum_{j=1}^{J} \sum_{i=1}^{m_{j}} \frac{m_{j}+1-i}{\eta_{j}} E^{u}\left[e^{-\theta \tau_{b}^{+}} \mathbf{1}_{K_{j i}}\right] \\
& +V(b ; b) \sum_{j=1}^{J} \sum_{i=1}^{m_{j}} E^{u}\left[e^{-\theta \tau_{b}^{+}} \mathbf{1}_{K_{j i}}\right] \\
& +E^{u}\left[e^{-\theta \tau_{b}^{+}} \mathbf{1}_{\left\{\tau_{b}^{+}<\tau_{u}\right\}} \mathbf{1}_{\left\{X_{\tau}=b\right\}} E^{X_{\tau_{b}^{+}}}\right. \\
& \left.\times\left(\int_{0}^{T} e^{-\theta t} d D(t) \cdot \theta_{\tau_{b}^{+}}\right)\right] \\
& =\sum_{j=1}^{J} \sum_{i=1}^{m_{j}} \frac{m_{j}+1-i}{\eta_{i}} E^{u}\left[e^{-\theta \tau_{b}^{+}} \mathbf{1}_{K_{j i}}\right]
\end{aligned}
$$




$$
\begin{aligned}
+V(b, b)\left(E^{u}\left[e^{-\theta \tau_{b}^{+}} \mathbf{1}_{K_{0}}\right]\right. \\
\left.+\sum_{j=1}^{J} \sum_{i=1}^{m_{j}} E^{u}\left[e^{-\theta \tau_{b}^{+}} \mathbf{1}_{K_{j i}}\right]\right) .
\end{aligned}
$$

Assertion (2) follows from (1) and $V^{\prime}(b ; b)=1$, and assertion (3) follows from (1) and $P\left(K_{0}\right)=0$. This ends the proof.

For threshold strategy, we assume that dividends are paid according to the following strategy governed by parameters $b>0$ and $\alpha>0$. Whenever the modified surplus is below the threshold level $b$, no dividends are paid. However, when the surplus is above this threshold level, dividends are paid at a constant rate $\alpha$ that does not exceed the premium rate $\mu>0$. We define the modified risk process $U_{b}=\left\{U_{b}(t): t \geq 0\right\}$ by $U_{b}(t)=X(t)-D_{b}(t)$, where

$$
D_{b}(t)=\alpha \int_{0}^{t} \mathbf{1}\left(U_{b}(s)>b\right) d s .
$$

Let $D_{b}$ denote the present value of all dividends until the time of ruin $\widetilde{T}_{2}$,

$$
D_{b}=\alpha \int_{0}^{\widetilde{T}_{2}} e^{-\theta t} d D_{b}(t),
$$

where $\widetilde{T}_{2}=\inf \left\{t>0: U_{b}(t)<0\right\}$ with $\widetilde{T}_{2}=\infty$ if $U_{b}(t)=0$ for all $t \geq 0$. Here $\theta>0$ is the discount factor. Denote by $V(u, b)$ the expected discounted value of dividend payments; that is, $V(u, b)=E\left[D_{b} \mid U_{b}(0)=u\right]$.

Similar to the proof of Theorem 8 , we have the following result.

Theorem 9. Let $\tau_{b}^{-}=\inf \{t \geq 0: X(t) \leq b\}, \tau_{b}^{+}=\inf \{t \geq$ $0: X(t) \geq b\}$, and $\tau=T \wedge \tau_{b}^{+}$. Then, for $\theta>0$, one has the following.

(1) for $u>b$,

$$
\begin{aligned}
V(u ; b)= & \frac{\alpha}{\theta}-\frac{\alpha}{\theta} E^{u}\left[e^{-\theta \tau_{b}^{-}} \mathbf{1}_{F_{0}}\right]-\frac{\alpha}{\theta} \sum_{j=1}^{\widehat{J}} \sum_{i=1}^{\widehat{m}_{j}} E^{u}\left[e^{-\theta \tau_{b}^{-}} \mathbf{1}_{F_{j i}}\right] \\
& +V(b, b) E^{u}\left[e^{-\theta \tau_{b}^{-}} \mathbf{1}_{F_{0}}\right]+\sum_{j=1}^{\widehat{J}} \sum_{i=1}^{\widehat{m}_{j}} E^{u}\left[e^{-\theta \tau_{b}^{-}} \mathbf{1}_{F_{j i}}\right] f_{j i} ;
\end{aligned}
$$

(2) for $b>u>0$, if $\mu>0$, then

$$
\begin{aligned}
V(u ; b)= & \alpha \sum_{j=1}^{J} \sum_{i=1}^{m_{j}} \frac{m_{j}+1-i}{\eta_{j}} E^{u}\left[e^{-\theta \tau_{b}^{+}} \mathbf{1}_{K_{j i}}\right] \\
& +V(b, b) E^{u}\left[e^{-\theta \tau_{b}^{+}} \mathbf{1}_{K_{0}}\right] \\
& +V(b, b) \sum_{j=1}^{J} \sum_{i=1}^{m_{j}} E^{u}\left[e^{-\theta \tau_{b}^{+}} \mathbf{1}_{K_{j i}}\right]
\end{aligned}
$$

(3) for $b>u>0$, if $\mu \leq 0$, then

$$
\begin{aligned}
V(u ; b)= & \alpha \sum_{j=1}^{J} \sum_{i=1}^{m_{j}} \frac{m_{j}+1-i}{\eta_{j}} E^{u}\left[e^{-\theta \tau_{b}^{+}} \mathbf{1}_{K_{j i}}\right] \\
& +V(b, b) \sum_{j=1}^{J} \sum_{i=1}^{m_{j}} E^{u}\left[e^{-\theta \tau_{b}^{+}} \mathbf{1}_{K_{j i}}\right],
\end{aligned}
$$

where $F_{0}=\left\{\omega: U_{b}\left(\tau_{b}^{-}\right)=b\right\}, F_{j i}=\left\{\omega: U_{b}\left(\tau_{b}^{-}\right)<\right.$ $\left.b,-Y_{N_{\tau_{b}^{-}}} \sim \operatorname{Erlang}\left(\widehat{\rho}_{j}, i\right)\right\}, j=1,2, \ldots, \widehat{J}, i=1,2, \ldots, \widehat{m}_{j}$, and $f_{j i}=\int_{-\infty}^{0} V(y+b ; b)\left(\left(-\widehat{\rho}_{j} y\right)^{i-1} \widehat{\rho}_{j} e^{\hat{\rho}_{j} y} /(i-1) !\right) d y$.

Remark 10. If $m_{j}=1, j=1,2, \ldots, J, \widehat{m}_{j}=1$, and $j=$ $1,2, \ldots, \widehat{J}$, that is, the process $X$ has the hyperexponential downward jumps and upward jumps, then we have the known results (Theorems 3.2 and 3.4, of Yin et al. [10]).

\section{Conflict of Interests}

The authors declare that there is no conflict of interests regarding the publication of this paper.

\section{Acknowledgments}

The authors cordially thank the editor and anonymous referee for their careful reading and helpful comments on the previous version of this paper, which led to a considerable improvement of the presentation of the work. This work was supported by the National Natural Science Foundation of China (no. 11171179 and no. 11301295), Natural Science Foundation of Shandong Province (no. ZR2010AQ015), and the Research Fund for the Doctoral Program of Higher Education of China (no. 20133705110002).

\section{References}

[1] D. Perry, W. Stadje, and S. Zacks, "First-exit times for compound Poisson processes for some types of positive and negative jumps," Stochastic Models, vol. 18, no. 1, pp. 139-157, 2002.

[2] S. G. Kou and H. Wang, "First passage times of a jump diffusion process," Advances in Applied Probability, vol. 35, no. 2, pp. 504531, 2003.

[3] N. Cai, "On first passage times of a hyper-exponential jump diffusion process," Operations Research Letters, vol. 37, no. 2, pp. 127-134, 2009.

[4] Y. Chi, "Analysis of the expected discounted penalty function for a general jump-diffusion risk model and applications in finance," Insurance, vol. 46, no. 2, pp. 385-396, 2010.

[5] T. Kadankova and N. Veraverbeke, "On several two-boundary problems for a particular class of lévy processes," Journal of Theoretical Probability, vol. 20, no. 4, pp. 1073-1085, 2007.

[6] D. Perry, W. Stadje, and S. Zacks, "Contributions to the theory of first-exit times of some compound processes in queueing theory," Queueing Systems, vol. 33, no. 4, pp. 369-379, 1999.

[7] A. L. Lewis and E. Mordecki, "Wiener-Hopf factorization for Ĺevy processes having positive jumps with rational transforms," Journal of Applied Probability, vol. 45, no. 1, pp. 118-134, 2008. 
[8] A. Kuznetsov, "On the distribution of exponential functionals for Lévy processes with jumps of rational transform," Stochastic Processes and their Applications, vol. 122, no. 2, pp. 654-663, 2012.

[9] Y. Chi and X. S. Lin, "On the threshold dividend strategy for a generalized jump-diffusion risk model," Insurance, vol. 48, no. 3, pp. 326-337, 2011.

[10] C. C. Yin, Y. Shen, and Y. Z. Wen, "Exit problems for jump processes with applications to dividend problems," Journal of Computational and Applied Mathematics, vol. 245, pp. 30-52, 2013. 


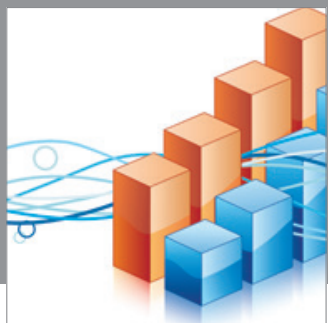

Advances in

Operations Research

mansans

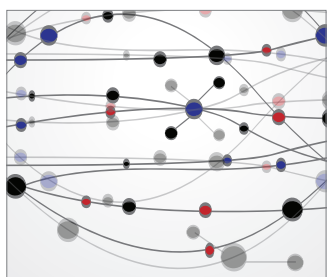

The Scientific World Journal
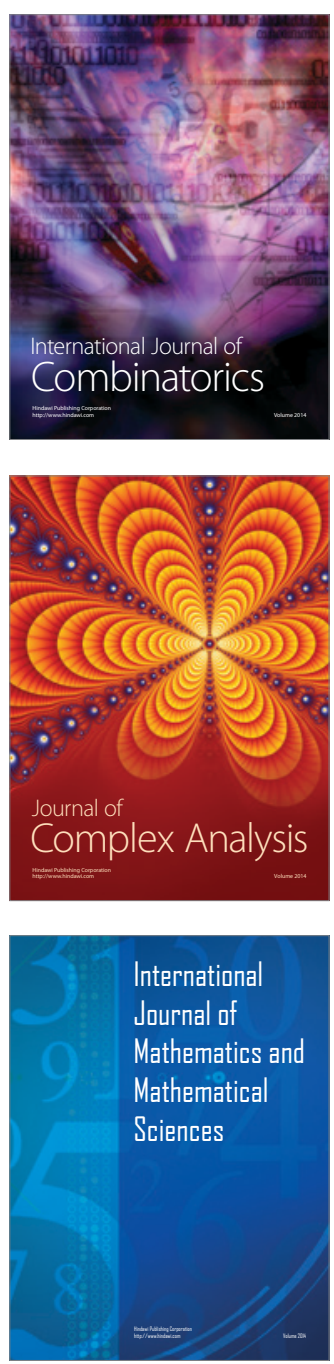
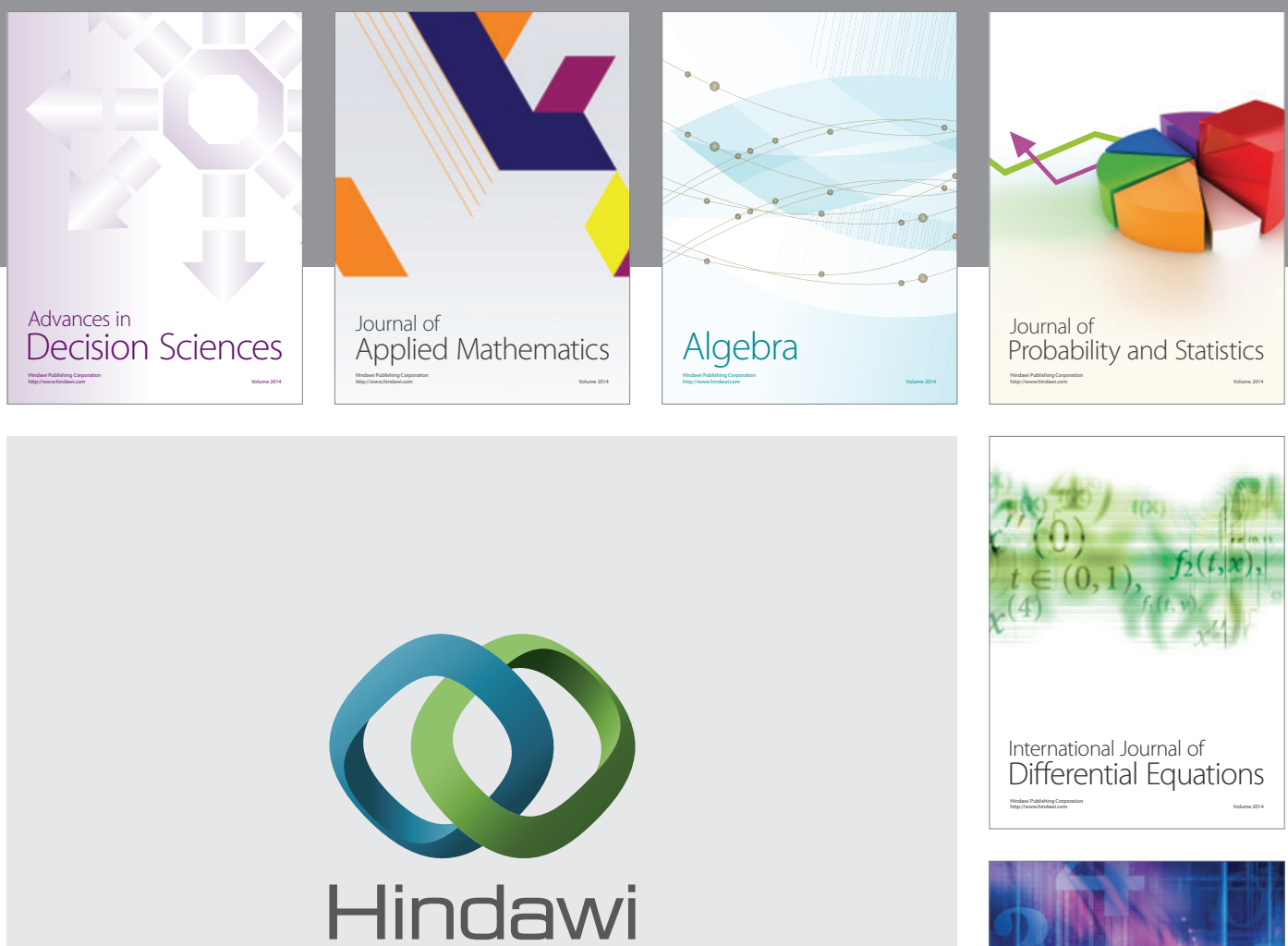

Submit your manuscripts at http://www.hindawi.com
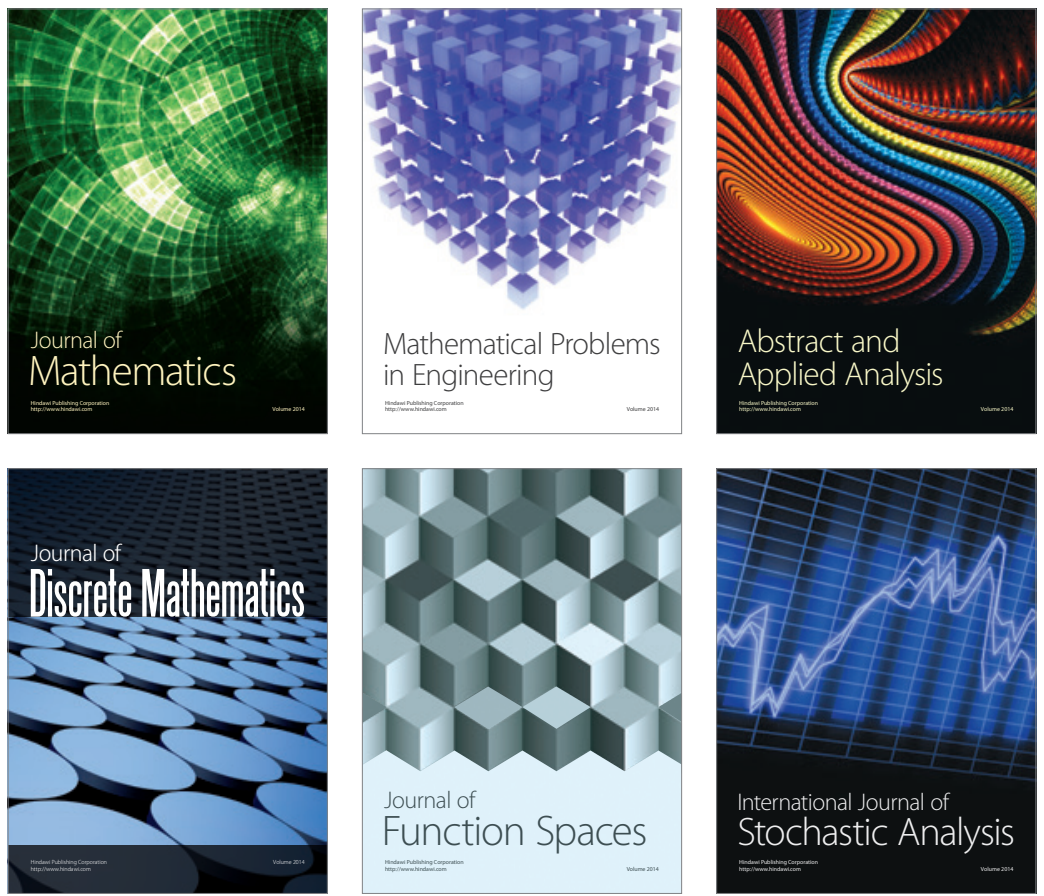

Journal of

Function Spaces

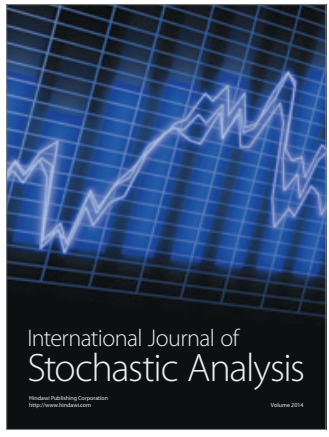

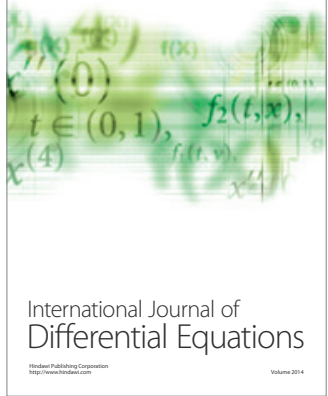
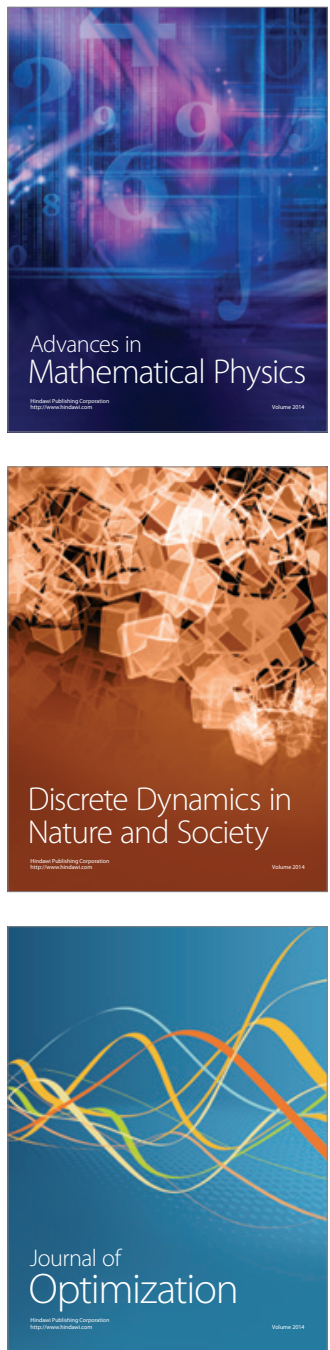